$(p=0.003$ and $p=0.005)$. In particular, there was an improvement in two SGRQ domains: activities $(p=0.013)$ and symptoms $(p=0.005)$ (fig.1).

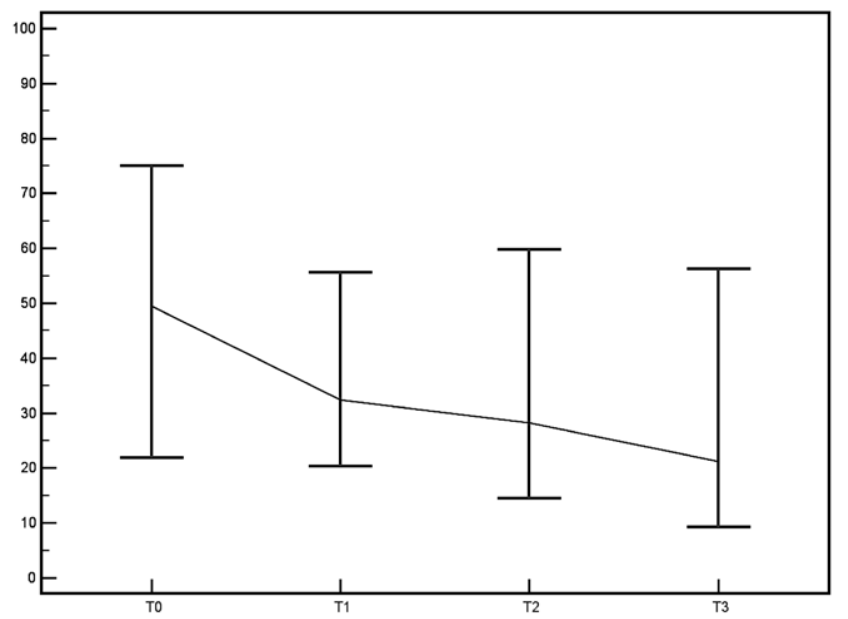

Figure 1

Conclusion: This is the first study to investigate the effect of EFA technology on airway clearance in SSc patients. The observations suggest the importance of a daily ACT program with EFA in improving respiratory symptoms. This technology appear to be extremely promising in SS patient management as it is well tolerated and it has the potential to slow down the pulmonary disease progression by limiting bronchial infections.

References:

[1] Tyndall AJ et al. Causes and risk factors for death in systemic sclerosis: a study from the EULAR Scleroderma Trials and Research (EUSTAR) database. Ann Rheum Dis 2010;69:1809-15.

[2] Zampogna E, et al. Expiratory Flow Accelerator (EFA) technique on mucus hypersecretion of COPD patients with reduced cough efficiency after a severe exacerbation. Int Clin Med 2019;3:1-6.

Disclosure of Interests: Silvia faverzani: None declared, Andrea Becciolini Speakers bureau: Sanofi-Genzyme, UCB and AbbVie, ernesto crisafulli: None declared, filippo nocera: None declared, eleonora di donato: None declared, Flavio Mozzani: None declared, michele riva: None declared, Daniele Santilli: None declared, lorenza monica: None declared, annalisa barbieri: None declared, lissette barone: None declared, maurizio marvisi: None declared, veronica alfieri: None declared, annalisa frizzelli: None declared, Alfredo Chetta: None declared, ALARICO ARIANI: None declared

DOI: 10.1136/annrheumdis-2020-eular.2434

\section{THU0617-HPR TOWARDS A UNIVERSAL DEFINITION OF DISEASE ACTIVITY SCORES THRESHOLDS}

N. Foulquier ${ }^{1}$, B. Chevet ${ }^{2}$, G. Carvajal Alegria ${ }^{1}$, L. Saraux ${ }^{3}$, V. DevauchellePensec $^{1}$, P. Redou ${ }^{1}$, A. Saraux ${ }^{1} .{ }^{1} \mathrm{CHU}$, Univ Brest and Inserm, Brest, France; ${ }^{2} \mathrm{CHU}$, Brest, France; ${ }^{2} \mathrm{CHU}$, Brest, France

Background: For rheumatologists monitoring patients with various diseases and dealing with multiple scores with different maximum values ( 9 for RA-DAS, 6.4 for AS-DAS and 60 for PMR-AS) and values thresholds to characterize the different levels of disease activity (low, intermediate and high) can be a tedious task. The same problematic could arise in other specialty than rheumatology. Normalization of these scores seems to be necessary to facilitate daily clinical practice (1).

Objectives: To indentify and standardize scores of activity of inflammatory diseases.

Methods: We conducted a literature review on activity criteria using both a manual approach and the BIBOT software (2) published in English between 1.1.1975 and 31.12.2018. Within all extracted disease activity scores, we selected those with cut off values in four classes (remission, low, moderate and high disease activity). We used a linear interpolation to map all these disease activity scores to our new score, the AS-135, and developed a smart-phone application to perform the conversion automatically.

Results: 1068 articles were analyzed by BIBOT, 86 were excluded on the basis of the language used for their writing and 11 were excluded on the basis of their publication date. 599 were selected based on their titles, abstracts and keywords. 108 activity criteria from various fields (rheumatology, dermatology, gastroenterology, psychiatry, neurology and pneumology) were identified, but it is in rheumatology that we find separation into four classes. 10 scores met our

inclusion criteria and were implemented in the Android app. These are: DAS28 (ESR), DAS28 (CRP), SDAI, ASDAS (ESR), ASDAS (CRP), ESSDAI, SLEDAI-2K, DAPSA, PMR-AS (ESR) and PMR-AS (CRP). We built the AS135 score modification for each selected score using a linear interpolation of the existing criteria. It was defined on the interval $[0,10]$ and values 1,3 and 5 were used as thresholds. These arbitrary thresholds are then associated with the thresholds of the existing criteria and an interpolation can be calculated, allowing the conversion of the existing criteria into AS135 criterion. We have finally created a mobile application that allows each user to obtain both the original value of the activity criterion.

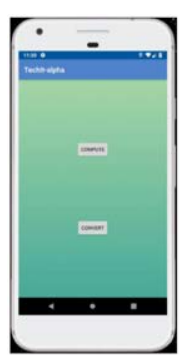

a)

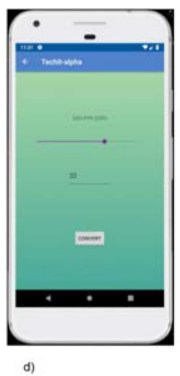

Conclusion: We have created a mobile application that allows any user to obtain in a simple way the level of disease activity, whatever the criterion used to describe it, since the application returns, in addition to the value of the activity criterion calculated from data returned by the physician, the transformation of this value into AS135 criterion and its interpretation in terms of level of activity of the pathology. The application is now available for Android devices and we plan to start developing a version for iOS devices.

\section{References:}

[1] Saraux L, Devauchelle-Pensec V, Saraux A. Plea for standardization of disease activity scores. Rheumatol Oxf Engl. 2019 Aug 1;58(8):1500-1

[2] Orgeolet L, Foulquier N, Misery L, Redou P, Pers J-O, Devauchelle-Pensec $\mathrm{V}$, et al. Can artificial intelligence replace manual search for systematic literature? Review on cutaneous manifestations in primary Sjögren's syndrome. Rheumatol Oxf Engl. 2019 Aug 31;

Disclosure of Interests: None declared

DOI: 10.1136/annrheumdis-2020-eular.1851

\section{THU0618-HPR PSYCHOSOCIAL CHANGES IN RHEUMATIC DISEASE: A NURSING LED CROSS-SECTIONAL STUDY}

R. Spagnuolo ${ }^{1}$, R. D. Grembiale ${ }^{2}$, F. S. laquinta ${ }^{2}$, V. Funari ${ }^{2}$, R. Pagnotta ${ }^{1}$, S. Naty ${ }^{2}$, P. Doldo ${ }^{1}{ }^{1}$ Magna Graecia University, School of Nursing, Catanzaro, Italy; ${ }^{2}$ Magna Graecia University, Rheumatology Research Unit, Catanzaro, Italy

Background: Nursing management in Rheumatic Diseases (RD) is focused on global patient care. Starting from basic knowledge of diagnostic and therapeutic management, nurses can assess the impact of RD on patients' quality of life not only at the physical level, but also at the psychological, social, and emotional levels.

Objectives: To evaluate psycosocial changes in RD patients through nursing-led Patient-Reported Outcomes

Methods: We performed a cross-sectional study of $100 \mathrm{RD}$ patients compared with 100 healthy volunteers matched for age, sex and BMI. Specialist nurses invited patients and volunteers to complete questionnaires on quality of life through seven domains (anxiety, depression, fatigue, sleep disturbance, pain interference, physical functions and satisfaction with participation in socia 
roles) of the Patient-Reported Outcomes Measurement Information System (PROMIS).

Results: Among $100 \mathrm{RD}$ patients, 52 (52\%) had a diagnosis of Rheumatoid Arthritis; 17 (17\%) had a diagnosis of axial spondylorthritis (Ankylosing Spondylitis and Psoriatic Artritis); 25 (25\%) had connectivitis (i.e. Lupus, Systemic Sclerosis, Sjögren Syndrome), and finally 6 (6\%) had vasculitis. Median disease duration was $7 \pm 5$ years. Just under half $(43 \%)$ of RD patients had active disease measured by specific disease activity index. As shown in table 1, no significant difference highlight between the two groups with regard to anthropompetric and demographic characteristics. We found that patients report significantly greater psychosocial changes than healthy controls. More specifically, as shown in figure $1 \mathrm{~A}$, mean $\mathrm{T}$ score for anxiety, depression, fatigue and sleep disturbances were significantly higher in the RD patients than in healthy controls $(56 \pm 9$ vs $48 \pm 8 p<0.001 ; 52 \pm 9$ vs $46 \pm 8 p<0.001 ; 58 \pm 8 v s 48 \pm 8 p<0.001 ; 52 \pm 10$ vs $44 \pm 8 p<0.001)$ respectively. Moreover, also in the social dimension in terms of pain interference, physical functions and satisfaction with participation in social roles, patients showed a median T score worse than healthy controls (Fig.1B).

Table 1

\begin{tabular}{lccc}
\hline & Patients (N=100) & Healthy $(\mathrm{N}=100)$ & $p$ \\
\hline Demographic and & Age (years) & $52.5 \pm 11$ & $51 \pm 18$ \\
Anthropometric & & & \\
ns & Gender n male (\%) & $43(43)$ & $47(47)$ \\
ns & BMl (Kg/m2) & $25.1 \pm 4$ & $27.8 \pm 4$ \\
ns & Smoke $\mathrm{n}(\%)$ & $52(52)$ & $46(46)$ \\
ns & $42(42)$ & $41(41)$ \\
ns & Marital Status n not married (\%) & $31(31)$ & $35(35)$ \\
ns & Occupation n yes (\%) & $54(54)$ & $64(64)$ \\
ns & Education level n degree (\%) & $52(52)$ & - \\
& Rheumatoid Arthritis & $17(17)$ & - \\
& Axial Spondylorthritis & $25(25)$ & - \\
& Connectivitis & $6(6)$ & - \\
& Vasculitis & $7.1 \pm 5.18$ & - \\
& Disease duration (years) & $43(43)$ & - \\
& Disease Activity n yes (\%) & $7(7)$ & - \\
& Medications n (\% patients) & $26(26)$ & - \\
& NSAlD & $54(54)$ & - \\
& Steroids & $34(34)$ & - \\
\hline
\end{tabular}

Continuous variables are shown as mean \pm standard deviation. Categorical variables are presented as number and proportion. The overall $p$-value was calculated by the Mann-Whitney non-parametric test for independent samples and by Chi-square test as appropriate

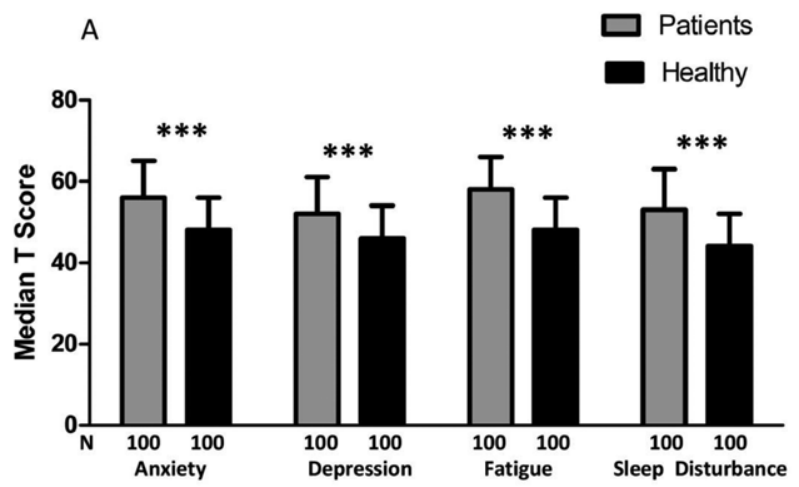

B

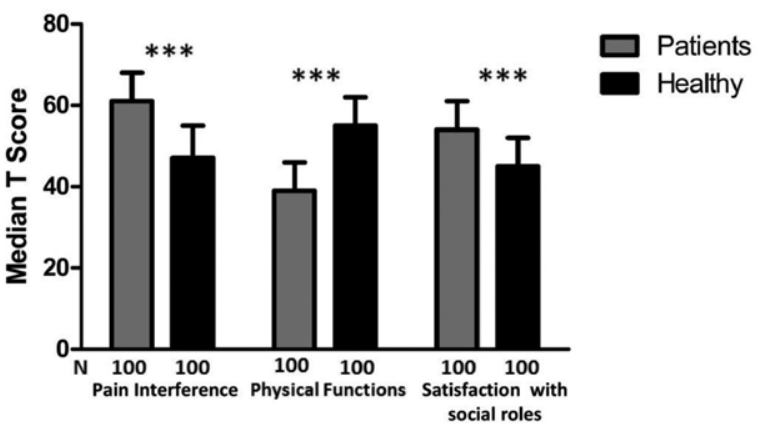

Figure 1. Median T Score stratified by study group. Data are shown as mean and standard deviation. The overall $p$-value wascalculated by the Mann-Whitney non-parametric test for independent samples.
Conclusion: This exploratory study highlights the need to adopt validated questionnaires in clinical practice, and demonstrates that PROMIS is a valid, objective, and standardized instrument that can help nursing staff to better define the consequences of the disease in a patient's daily life.

References:

[1] Minnock P, McKee G, Kelly A, et al. Nursing sensitive outcomes in patients with rheumatoid arthritis: a systematic literature review.Int. J. Nurs. Stud., 77 (2017), pp. 115-129

[2] Bartlett SJ, Orbai AM, Duncan T, et al. Reliability and validity of selected PROMIS measures in people with rheumatoid arthritis. PloS One. 2015

Disclosure of Interests: None declared

DOI: 10.1136/annrheumdis-2020-eular.4493

\section{THU0619-HPR PREVALENCE OF DISTAL INTERPHALANGEAL JOINT ULTRASONOGRAPHY FEATURES IN PSORIATIC ARTHRITIS, SKIN PSORIASIS, OSTEOARTHRITIS AND HEALTHY INDIVIDUALS: A CROSS-SECTIONAL STUDY}

J. Guldberg-Møller ${ }^{1,2}$, M. Henriksen ${ }^{1}$, M. Boesen $^{3}$, L. Dreyer ${ }^{4}$, K. Ellegaard ${ }^{1}$, M. Skougaard ${ }^{1}$, C. Ballegaard ${ }^{1}$, A. L. Tan ${ }^{5}$, R. Wakefield ${ }^{5}$, L. E. Kristensen ${ }^{1}$. ${ }^{1}$ Bispebjerg and Frederiksberg Hospital, The Parker Institute, Frederiksberg, Denmark; ${ }^{2}$ Slagelse Sygehus, Slagelse, Denmark; ${ }^{3}$ Bispebjerg and Frederiksberg Hospital, Department of Radiology, Copenhagen, Denmark; ${ }^{4}$ Aalborg University Hospital, Department of Rheumatology, Aalborg, Denmark; ${ }^{5}$ Leeds Institute of Rheumatic and Musculoskeletal Medicine, Leeds, United Kingdom

Background: Distal interphalangeal (DIP) joint involvement is a feature of both psoriatic arthritis (PsA) and hand osteoarthritis (OA), and nail-changes are features seen both in PsA and nail psoriasis patients without joint involvement (PsO). In both PsA and OA, ultrasonography (US) is used to quantify DIP joint inflammation. Objectives: To explore disease-specific US-detected characteristics in the DIPjoints and extensor tendon entheses in patients with DIP-joint OA, PsA, PsO with nail involvement, and healthy controls $(\mathrm{HC})$.

Methods: In PsA, PsO, OA and HC US examination of DIP joints 2-5 and the extensor tendon were performed. The US images were scored for DIP joint grey-scale synovitis, DIP joint Doppler, osteophytes and erosions (grade 0-3) and presence/absence of enthesitis and peritendonitis of the extensor tendon according to OMERACT standards. Prevalences were calculated on all included fingers (i.e. four fingers per participant), and differences in prevalences were tested using Chi-square statistics.

Results: Fifty PsA patients (44\% females; mean age: $55 \mathrm{y}$ ), $13 \mathrm{PsO}$ patients (38\% females; mean age $54 \mathrm{y}$ ), 12 OA patients ( $100 \%$ females, mean age $71 \mathrm{y})$ and $29 \mathrm{HC}$ (52\% females, mean age 48y) participated. The prevalences across the diagnosis groups are shown in figure 1, and the distribution of US outcomes was significantly different (highest Chi-square P-value: 0.0127 ). The PsA group had the largest prevalence of extensor tendon enthesitis $(45.5 \%)$, peritendonitis (15\%), and DIP joint erosions (11\%), but also exhibited a considerable prevalence of osteophytes (46\%). In the PsO group, the most marked findings were synovitis (33\%) and enthesitis (35\%). The OA group had the largest prevalence of DIP joint synovitis $(67 \%)$ and osteophytes $(88 \%)$ but also $25 \%$ prevalence of enthesitis. $24 \%$ of the $\mathrm{HC}$ group had a grade 1 synovitis.

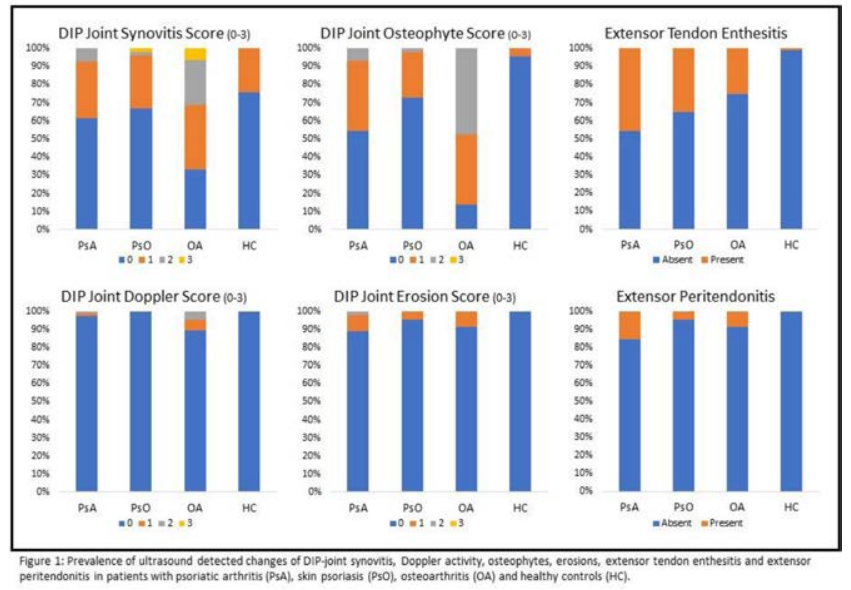

Conclusion: This cross-sectional study found significant patterns of US findings distributed dependent on the underlying condition. PsA patients were mainly differentiated by the presence of extensor tendon enthesitis and peritendonitis. A high prevalence of enthesitis and synovitis was seen in patients 